\title{
Berezin number inequalities for operators
}

https://doi.org/10.1515/conop-2019-0003

Received April 5, 2018; accepted February 5, 2019

Abstract: The Berezin transform $\widetilde{A}$ of an operator $A$, acting on the reproducing kernel Hilbert space $\mathscr{H}=$ $\mathscr{H}(\Omega)$ over some (non-empty) set $\Omega$, is defined by $\widetilde{A}(\lambda)=\left\langle A \hat{k}_{\lambda}, \hat{k}_{\lambda}\right\rangle(\lambda \in \Omega)$, where $\hat{k}_{\lambda}=\frac{k_{\lambda}}{\left\|k_{\lambda}\right\|}$ is the normalized reproducing kernel of $\mathscr{H}$. The Berezin number of an operator $A$ is defined by $\operatorname{ber}(A)=\sup _{\lambda \in \Omega}|\tilde{A}(\lambda)|=$ $\sup _{\lambda \in \Omega}\left|\left\langle A \hat{k}_{\lambda}, \hat{k}_{\lambda}\right\rangle\right|$. In this paper, we prove some Berezin number inequalities. Among other inequalities, it is shown that if $A, B, X$ are bounded linear operators on a Hilbert space $\mathscr{H}$, then

$$
\operatorname{ber}(A X \pm X A) \leqslant \operatorname{ber}^{\frac{1}{2}}\left(A^{\star} A+A A^{\star}\right) \operatorname{ber}^{\frac{1}{2}}\left(X^{\star} X+X X^{\star}\right)
$$

and

$$
\operatorname{ber}^{2}\left(A^{\star} X B\right) \leqslant\|X\|^{2} \operatorname{ber}\left(A^{\star} A\right) \operatorname{ber}\left(B^{\star} B\right) .
$$

We also prove the multiplicative inequality

$$
\operatorname{ber}(A B) \leqslant \operatorname{ber}(A) \operatorname{ber}(B) .
$$

Keywords: Reproducing kernel, Berezin number, Berezin transform.

MSC: Primary: 47A30, Secondary: 15A60, 30E20, 47A12, 47B15, 47B20

\section{Introduction}

Let $\mathscr{H}$ be a complex Hilbert space and $\mathbb{B}(\mathscr{H})$ denote the $C^{\star}$-algebra of all bounded linear operators on $\mathscr{H}$ with the identity $I$. In the case when $\operatorname{dim} \mathscr{H}=n$, we identify $\mathbb{B}(\mathscr{H})$ with the matrix algebra $\mathbb{M}_{n}$ of all $n \times n$ matrices having entries in the complex field $\mathbb{C}$. The numerical range and numerical radius of $A \in \mathbb{B}(\mathscr{H})$ are defined by $W(A):=\{\langle A x, x\rangle: x \in \mathscr{H},\|x\|=1\}$ and $w(A):=\sup \{|\lambda|: \lambda \in W(A)\}$, respectively. It is well known that $w(\cdot)$ defines a norm on $\mathbb{B}(\mathscr{H})$, which is equivalent to the usual operator norm $\|\cdot\|$. In fact, for any $A \in \mathbb{B}(\mathscr{H}), \frac{1}{2}\|A\| \leqslant w(A) \leqslant\|A\|$ (see [9, p. 9]).

A functional Hilbert space is a Hilbert space $\mathscr{H}=\mathscr{H}(\Omega)$ of complex-valued functions on a (non-empty) set $\Omega$, which has the property that point evaluations are continuous, i.e., for each $\lambda \in \Omega$ the map $f \longmapsto f(\lambda)$ is a continuous (linear) functional on $\mathscr{H}$. Then the Riesz representation theorem ensures that for each $\lambda \in \Omega$ there is a unique element $k_{\lambda}$ of $\mathscr{H}$ such that $f(\lambda)=\left\langle f, k_{\lambda}\right\rangle$ for all $f \in \mathscr{H}$. The collection $\left\{k_{\lambda}: \lambda \in \Omega\right\}$ is called the reproducing kernel of $\mathscr{H}$. If $\left\{e_{n}\right\}$ is an orthonormal basis for a functional Hilbert space $\mathscr{H}$, then the reproducing kernel of $\mathscr{H}$ is given by $k_{\lambda}(z)=\sum_{n} \overline{e_{n}(\lambda)} e_{n}(z)$. For $\lambda \in \Omega$, let $\hat{k}_{\lambda}=\frac{k_{\lambda}}{\left\|k_{\lambda}\right\|}$ be the normalized reproducing kernel of $\mathscr{H}$. For a bounded linear operator $A$ on $\mathscr{H}$, the function $\widetilde{A}$ defined on $\Omega$ by $\widetilde{A}(\lambda)=$ $\left\langle A \hat{k}_{\lambda}, \hat{k}_{\lambda}\right\rangle$, is the Berezin transform of $A$, which was introduced by Berezin [4, 5]. The Berezin set and the Berezin number of the operator $A$ are defined by (see [12])

$$
\operatorname{Ber}(A):=\{\widetilde{A}(\lambda): \lambda \in \Omega\} \text { and } \operatorname{ber}(A):=\sup \{|\widetilde{A}(\lambda)|: \lambda \in \Omega\},
$$

Mojtaba Bakherad: Department of Mathematics, Faculty of Mathematics, University of Sistan and Baluchestan, Zahedan, I.R.Iran, E-mail: mojtaba.bakherad@gmail.com; bakherad@member.ams.org

*Corresponding Author: Mubariz T. Garayev: Department of Mathematics, College of Science, King Saud University, P.O.Box 2455, Riyadh 11451, Saudi Arabia, E-mail: mgarayev@ksu.edu.sa 
respectively. It is clear that the Berezin transform $\widetilde{A}$ is the bounded function on $\Omega$ whose values lies in the numerical range of the operator $A$ and hence $\operatorname{Ber}(A) \subseteq W(A)$ and $\operatorname{ber}(A) \leqslant w(A)$ for all $A \in \mathbb{B}(\mathscr{H})$. Karaev [16] showed that for $A=S \otimes S \in \mathbb{B}\left(\mathscr{H}^{2}\right)$, where $S$ is the shift operator defined by $S f(z)=z f(z)$ on the HardyHilbert space $\mathscr{H}^{2}=\mathscr{H}^{2}(\mathbb{D})$ over the unit disc $\mathbb{D}=\{z \in \mathbb{C}:|z|<1\}$, we have $\widetilde{A}(\lambda)=|\lambda|^{2}\left(1-|\lambda|^{2}\right)$, and thus $\operatorname{Ber}(A)=\left[0, \frac{1}{4}\right] \varsubsetneqq[0,1]=W(A)$ and $\operatorname{ber}(A)=\frac{1}{4}<1=w(A)$. Moreover, the Berezin number of an operator $A$ satisfies the following properties:

(i) $\operatorname{ber}(A) \leqslant\|A\|$.

(ii) $\operatorname{ber}(\alpha A)=|\alpha| \operatorname{ber}(A)$ for all $\alpha \in \mathbb{C}$.

(iii) $\operatorname{ber}(A+B) \leqslant \operatorname{ber}(A)+\operatorname{ber}(B)$.

The Berezin transform has been investigated in detail for the Toeplitz and Hankel operators on the Hardy and Bergman spaces. It is widely applied in the various questions of analysis and uniquely determines the operator acting on the reproducing kernel Hilbert space of analytic functions in some suitable set $\Omega$ (i.e., $\widetilde{A}(\lambda)=\widetilde{B}(\lambda)$ for all $\lambda \in \Omega$ implies $A=B$ ). For further information about the Berezin transform and Berezin number we refer the reader to $[6-8,13-18,20-22,24]$ and references therein.

In this paper, by using some ideas of $[1,2,23]$, we present several Berezin number inequalities. In particular, we show that

Theorem: Let $A, B, X, Y \in \mathbb{B}(\mathscr{H})$. Then

(i) $\operatorname{ber}(A X \pm X A) \leqslant \operatorname{ber}^{\frac{1}{2}}\left(A^{\star} A+A A^{\star}\right) \operatorname{ber}^{\frac{1}{2}}\left(X^{\star} X+X X^{\star}\right)$;

(ii) $\operatorname{ber}\left(A^{\star} X B+B^{\star} Y A\right) \leqslant 2 \sqrt{\|X\|\|Y\|} \operatorname{ber}^{\frac{1}{2}}\left(B^{\star} B\right) \operatorname{ber}^{\frac{1}{2}}\left(A A^{\star}\right)$.

\section{Some Upper bounds for ber $\left(A X \pm X A^{\star}\right)$}

To prove our first result, we need the following lemma.

Lemma 2.1. Let $X \in \mathbb{B}(\mathscr{H})$. Then

$$
\operatorname{ber}(X)=\sup _{\theta \in \mathbb{R}} \operatorname{ber}\left(\mathfrak{R e}\left(e^{i \theta} X\right)\right)=\sup _{\theta \in \mathbb{R}} \operatorname{ber}\left(\mathfrak{I m}\left(e^{i \theta} X\right)\right),
$$

where $\mathfrak{R e}(X)=\frac{X+X^{\star}}{2}$ and $\mathfrak{I m}(X)=\frac{X-X^{*}}{2 i}$.

Proof. It follows from (see [23])

$$
\sup _{\theta \in \mathbb{R}}\left\langle\mathfrak{R e}\left(e^{i \theta} X\right) \hat{k}_{\lambda}, \hat{k}_{\lambda}\right\rangle=\left|\left\langle X \hat{k}_{\lambda}, \hat{k}_{\lambda}\right\rangle\right|
$$

that

$$
\begin{aligned}
\sup _{\theta \in \mathbb{R}} \operatorname{ber}\left(\mathfrak{R e}\left(e^{i \theta} X\right)\right) & =\sup _{\theta \in \mathbb{R}} \sup _{\lambda \in \Omega}\left|\left\langle\mathfrak{R e}\left(e^{i \theta} X\right) \hat{k}_{\lambda}, \hat{k}_{\lambda}\right\rangle\right| \\
& =\sup _{\lambda \in \Omega} \sup _{\theta \in \mathbb{R}}\left|\left\langle\mathfrak{R e}\left(e^{i \theta} X\right) \hat{k}_{\lambda}, \hat{k}_{\lambda}\right\rangle\right| \\
& =\sup _{\lambda \in \Omega}\left|\left\langle X \hat{k}_{\lambda}, \hat{k}_{\lambda}\right\rangle\right| \\
& =\operatorname{ber}(X) .
\end{aligned}
$$

If we replace $X$ by $i X$ in the first equation, we get the second equation. 
Remark 2.2. If $X=L+i K$ is the cartesian decomposition of the operator $X$, i.e., $L=\frac{X+X^{*}}{2}$ and $K=\frac{X-X^{*}}{2 i}$, then using this fact

$$
|\langle L x, x\rangle| \leqslant|\langle X x, x\rangle|=|\langle L x, x\rangle+i\langle K x, x\rangle|=\sqrt{|\langle L x, x\rangle|^{2}+\left.\langle K x, x\rangle\right|^{2}} \quad(x \in \mathscr{H}),
$$

and we have

$$
\operatorname{ber}(L)=\operatorname{ber}(\mathfrak{R e}(X)) \leqslant \operatorname{ber}(X) \leqslant \sqrt{\operatorname{ber}^{2}(L)+\operatorname{ber}^{2}(K)} .
$$

Now, by applying Lemma 2.1, we show an upper bound for $\operatorname{ber}\left(A X \pm X A^{\star}\right)$.

Theorem 2.3. Let $A, X \in \mathbb{B}(\mathscr{H})$. Then

$$
\begin{aligned}
& \operatorname{ber}^{2}\left(A X \pm X A^{\star}\right) \\
& \leqslant 2\|A\|^{2}\left(\operatorname{ber}\left(L^{2}\right)+\operatorname{ber}\left(K^{2}\right)+\sqrt{\left(\operatorname{ber}\left(L^{2}\right)-\operatorname{ber}\left(K^{2}\right)\right)^{2}+\operatorname{ber}^{2}(L K+K L)}\right),
\end{aligned}
$$

where $X=L+i K$ is the cartesian decomposition of the operator $X$.

Proof. By using the simple identity $\mathfrak{R e}\left(A B+B A^{\star}\right)=A \mathfrak{R e}(B)+\mathfrak{R e}(B) A^{\star}$ we have

$$
\begin{aligned}
& \left|\left\langle\mathfrak{R e}\left(e^{i \theta}\left(A X+X A^{\star}\right)\right) \hat{k}_{\lambda}, \hat{k}_{\lambda}\right\rangle\right|^{2} \leqslant\left|\left\langle\left(A \mathfrak{R e}\left(e^{i \theta} X\right)+\mathfrak{R e}\left(e^{i \theta} X\right) A^{\star}\right) \hat{k}_{\lambda}, \hat{k}_{\lambda}\right\rangle\right|^{2} \\
& \text { (since }|\langle\mathfrak{R e}(T) x, x\rangle| \leqslant|\langle T x, x\rangle| \text { ) } \\
& \leqslant 2\left(\left|\left\langle A \Re \mathfrak{R e}\left(e^{i \theta} X\right) \hat{k}_{\lambda}, \hat{k}_{\lambda}\right\rangle\right|^{2}+\left|\left\langle\mathfrak{R e}\left(e^{i \theta} X\right) A^{\star} \hat{k}_{\lambda}, \hat{k}_{\lambda}\right\rangle\right|^{2}\right) \\
& \text { (by the triangle inequality and the convexity } f(t)=t^{2} \text { ) } \\
& =2\left(\left|\left\langle\mathfrak{R e}\left(e^{i \theta} X\right) \hat{k}_{\lambda}, A^{\star} \hat{k}_{\lambda}\right\rangle\right|^{2}+\left|\left\langle A^{\star} \hat{k}_{\lambda}, \mathfrak{R e}\left(e^{i \theta} X\right) \hat{k}_{\lambda}\right\rangle\right|^{2}\right) \\
& \leqslant 2\left(\left\|A^{\star}\right\|^{2}\left\|\operatorname{Re}\left(e^{i \theta} X\right) \hat{k}_{\lambda}\right\|^{2}+\left\|A^{\star}\right\|^{2}\left\|\mathfrak{R e}\left(e^{i \theta} X\right) \hat{k}_{\lambda}\right\|^{2}\right) \\
& =4\|A\|^{2}\left\langle\mathfrak{R e}\left(e^{i \theta} X\right) \hat{k}_{\lambda}, \mathfrak{R e}\left(e^{i \theta} X\right) \hat{k}_{\lambda}\right\rangle \\
& =4\|A\|^{2}\left\langle\left(\Re \mathfrak{R e}\left(e^{i \theta} X\right)\right)^{2} \hat{k}_{\lambda}, \hat{k}_{\lambda}\right\rangle \text {. }
\end{aligned}
$$

It follows from

$$
\begin{aligned}
\left(\mathfrak{R e}\left(e^{i \theta} X\right)\right)^{2} & =\left(\mathfrak{R e}\left(e^{i \theta}(L+i K)\right)\right)^{2} \\
& =(\cos \theta L-\sin \theta K)^{2} \\
& =\cos ^{2} \theta L^{2}+\sin ^{2} \theta K^{2}-\cos \theta \sin \theta(L K+K L)
\end{aligned}
$$

that

$$
\begin{aligned}
& \sup _{\theta \in \mathbb{R}}\left\langle\left(\mathfrak{R e}\left(e^{i \theta} X\right)\right)^{2} \hat{k}_{\lambda}, \hat{k}_{\lambda}\right\rangle \\
& =\sup _{\theta \in \mathbb{R}}\left(\cos ^{2} \theta\left\langle L^{2} \hat{k}_{\lambda}, \hat{k}_{\lambda}\right\rangle+\sin ^{2} \theta\left\langle K^{2} \hat{k}_{\lambda}, \hat{k}_{\lambda}\right\rangle-\cos \theta \sin \theta\left(\left\langle(L K+K L) \hat{k}_{\lambda}, \hat{k}_{\lambda}\right\rangle\right)\right) \\
& \leqslant \sup _{\theta \in \mathbb{R}}\left(\cos ^{2} \theta \operatorname{ber}\left(L^{2}\right)+\sin ^{2} \theta \operatorname{ber}\left(K^{2}\right)-\cos \theta \sin \theta\left(\left\langle(L K+K L) \hat{k}_{\lambda}, \hat{k}_{\lambda}\right\rangle\right)\right) \\
& \leqslant \frac{1}{2}\left(\operatorname{ber}\left(L^{2}\right)+\operatorname{ber}\left(K^{2}\right)+\sqrt{\left(\operatorname{ber}\left(L^{2}\right)-\operatorname{ber}\left(K^{2}\right)\right)^{2}+\left(\left\langle(L K+K L) \hat{k}_{\lambda}, \hat{k}_{\lambda}\right\rangle\right)^{2}}\right),
\end{aligned}
$$

whence

$$
\begin{aligned}
& \sup _{\theta \in \mathbb{R}}\left|\left\langle\mathfrak{R e}\left(e^{i \theta}\left(A X+X A^{\star}\right)\right) \hat{k}_{\lambda}, \hat{k}_{\lambda}\right\rangle\right|^{2} \\
& \leqslant 2\|A\|^{2}\left(\operatorname{ber}\left(L^{2}\right)+\operatorname{ber}\left(K^{2}\right)+\sqrt{\left(\operatorname{ber}\left(L^{2}\right)-\operatorname{ber}\left(K^{2}\right)\right)^{2}+\left(\left\langle(L K+K L) \hat{k}_{\lambda}, \hat{k}_{\lambda}\right\rangle\right)^{2}}\right) .
\end{aligned}
$$


Taking the supremum over all $\lambda \in \Omega$ and using Lemma 2.1, we get

$$
\begin{aligned}
\operatorname{ber}^{2}\left(A X+X A^{\star}\right) & =\sup _{\theta \in \mathbb{R}} \operatorname{ber}\left(\Re \mathfrak{R e}\left(e^{i \theta}\left(A X+X A^{\star}\right)\right)\right) \\
& \leqslant 2\|A\|^{2}\left(\operatorname{ber}\left(L^{2}\right)+\operatorname{ber}\left(K^{2}\right)+\sqrt{\left(\operatorname{ber}\left(L^{2}\right)-\operatorname{ber}\left(K^{2}\right)\right)^{2}+\operatorname{ber}^{2}(L K+K L)}\right) .
\end{aligned}
$$

Replacing $A$ by $i A$ in (2.1), we get

$$
\operatorname{ber}^{2}\left(A X-X A^{\star}\right) \leqslant 2\|A\|^{2}\left(\operatorname{ber}\left(L^{2}\right)+\operatorname{ber}\left(K^{2}\right)+\sqrt{\left(\operatorname{ber}\left(L^{2}\right)-\operatorname{ber}\left(K^{2}\right)\right)^{2}+\operatorname{ber}^{2}(L K+K L)}\right) .
$$

Hence

$$
\operatorname{ber}^{2}\left(A X \pm X A^{\star}\right) \leqslant 2\|A\|^{2}\left(\operatorname{ber}\left(L^{2}\right)+\operatorname{ber}\left(K^{2}\right)+\sqrt{\left(\operatorname{ber}\left(L^{2}\right)-\operatorname{ber}\left(K^{2}\right)\right)^{2}+\operatorname{ber}^{2}(L K+K L)}\right)
$$

as required.

Theorem 2.3 includes a special case as follows.

Corollary 2.4. Let $A, X \in \mathbb{B}(\mathscr{H})$ Then

(i) If $L K+K L=0$, then $\operatorname{ber}\left(A X \pm X A^{\star}\right) \leqslant 2\|A\| \max \left(\operatorname{ber}^{\frac{1}{2}}\left(L^{2}\right), \operatorname{ber}^{\frac{1}{2}}\left(K^{2}\right)\right)$.

(ii) If $X$ is self-adjoint, then $\operatorname{ber}\left(A X \pm X A^{\star}\right) \leqslant 2\|A\|$ ber $^{\frac{1}{2}}\left(X^{2}\right)$.

(iii) If $X$ is self-adjoint, then $\operatorname{ber}(A X) \leqslant\|A\| \operatorname{ber}^{\frac{1}{2}}\left(X^{2}\right)$.

where $X=L+i K$ is the cartesian decomposition of the operator $X$.

Proof. The first inequality follows from Theorem 2.3 and the inequality

$$
\begin{aligned}
\operatorname{ber}^{2}\left(A X \pm X A^{\star}\right) & \leqslant 2\|A\|^{2}\left(\operatorname{ber}\left(L^{2}\right)+\operatorname{ber}\left(K^{2}\right)+\sqrt{\left(\operatorname{ber}\left(L^{2}\right)-\operatorname{ber}\left(K^{2}\right)\right)^{2}}\right) \\
& =2\|A\|^{2}\left(\operatorname{ber}\left(L^{2}\right)+\operatorname{ber}\left(K^{2}\right)+\left|\operatorname{ber}\left(L^{2}\right)-\operatorname{ber}\left(K^{2}\right)\right|\right) \\
& =4\|A\|^{2} \max \left(\operatorname{ber}\left(L^{2}\right), \operatorname{ber}\left(K^{2}\right)\right) .
\end{aligned}
$$

The second inequality follows from Theorem 2.3 and the hypotheses $X=L+0 i$. For the third inequality we have

$$
\begin{aligned}
\operatorname{ber}(A X) & =\sup _{\theta \in \mathbb{R}} \operatorname{ber}\left(\mathfrak{R e}\left(e^{i \theta} A X\right)\right) \\
& =\frac{1}{2} \sup _{\theta \in \mathbb{R}} \mathbf{b e r}\left(e^{i \theta} A X+e^{-i \theta} X A^{\star}\right) \\
& \leqslant\|A\| \operatorname{ber}^{\frac{1}{2}}\left(X^{2}\right) \quad \text { (by part (ii)), }
\end{aligned}
$$

as required.

\section{Upper bounds for $\operatorname{ber}(A X \pm X A)$ and $\operatorname{ber}\left(A^{\star} X B \pm B^{\star} Y A\right)$}

The following theorem gives some upper bounds for $\operatorname{ber}(A X \pm X A)$.

Theorem 3.1. Let $A, X \in \mathbb{B}(\mathscr{H})$. Then

$$
\begin{aligned}
& \text { (i) } \operatorname{ber}(A X \pm X A) \leqslant \operatorname{ber}^{\frac{1}{2}}\left(A^{\star} A+A A^{\star}\right) \operatorname{ber}^{\frac{1}{2}}\left(X^{\star} X+X X^{\star}\right) . \\
& \text { (ii) } \operatorname{ber}(A X \pm X A) \leqslant \operatorname{ber}^{\frac{1}{2}}\left(A^{\star} A+X^{\star} X\right) \operatorname{ber}^{\frac{1}{2}}\left(A A^{\star}+X X^{\star}\right) \text {. }
\end{aligned}
$$


Proof. Let $\hat{k}_{\lambda}$ be the normalized reproducing kernel of $\mathscr{H}$. Then

$$
\begin{aligned}
\left|\left\langle(A X \pm X A) \hat{k}_{\lambda}, \hat{k}_{\lambda}\right\rangle\right| & \leqslant\left|\left\langle A X \hat{k}_{\lambda}, \hat{k}_{\lambda}\right\rangle\right|+\left|\left\langle X A \hat{k}_{\lambda}, \hat{k}_{\lambda}\right\rangle\right| \\
& =\left|\left\langle X \hat{k}_{\lambda}, A^{\star} \hat{k}_{\lambda}\right\rangle\right|+\left|\left\langle A \hat{k}_{\lambda}, X^{\star} \hat{k}_{\lambda}\right\rangle\right| \\
& \leqslant\left\|X \hat{k}_{\lambda}\right\|\left\|A^{\star} \hat{k}_{\lambda}\right\|+\left\|A \hat{k}_{\lambda}\right\|\left\|X^{\star} \hat{k}_{\lambda}\right\| \\
& \leqslant\left(\left\|A \hat{k}_{\lambda}\right\|^{2}+\left\|A^{\star} \hat{k}_{\lambda}\right\|^{2}\right)^{\frac{1}{2}}\left(\left\|X \hat{k}_{\lambda}\right\|^{2}+\left\|X^{\star} \hat{k}_{\lambda}\right\|^{2}\right)^{\frac{1}{2}}
\end{aligned}
$$

(by the Cauchy-Schwartz inequality)

$$
\begin{aligned}
& =\left|\left\langle\left(A^{\star} A+A A^{\star}\right) \hat{k}_{\lambda}, \hat{k}_{\lambda}\right\rangle\right|^{\frac{1}{2}}\left|\left\langle\left(X^{\star} X+X X^{\star}\right) \hat{k}_{\lambda}, \hat{k}_{\lambda}\right\rangle\right|^{\frac{1}{2}} \\
& \leqslant \operatorname{ber}^{\frac{1}{2}}\left(A^{\star} A+A A^{\star}\right) \operatorname{ber}^{\frac{1}{2}}\left(X^{\star} X+X X^{\star}\right) .
\end{aligned}
$$

Hence

$$
\begin{aligned}
\operatorname{ber}(A X \pm X A) & =\sup _{\lambda \in \mathbb{R}}\left|\left\langle(A X \pm X A) \hat{k}_{\lambda}, \hat{k}_{\lambda}\right\rangle\right| \\
& \leqslant \operatorname{ber}^{\frac{1}{2}}\left(A^{\star} A+A A^{\star}\right) \operatorname{ber}^{\frac{1}{2}}\left(X^{\star} X+X X^{\star}\right) .
\end{aligned}
$$

Now, according to the inequality

$$
\left\|X \hat{k}_{\lambda}\right\|\left\|A^{\star} \hat{k}_{\lambda}\right\|+\left\|A \hat{k}_{\lambda}\right\|\left\|X^{\star} \hat{k}_{\lambda}\right\| \leqslant\left(\left\|A \hat{k}_{\lambda}\right\|^{2}+\left\|X \hat{k}_{\lambda}\right\|^{2}\right)^{\frac{1}{2}}\left(\left\|A^{\star} \hat{k}_{\lambda}\right\|^{2}+\left\|X^{\star} \hat{k}_{\lambda}\right\|^{2}\right)^{\frac{1}{2}}
$$

(by the Cauchy-Schwartz inequality)

and a similar argument of the proof of part (i), we get the second inequality.

For the special case $A=I$, we have the next result.

Corollary 3.2. Let $X \in \mathbb{B}(\mathscr{H})$. Then

$$
\begin{aligned}
& \text { (i) } \operatorname{ber}^{2}(X) \leqslant \frac{1}{4} \operatorname{ber}\left(I+X^{\star} X\right) \operatorname{ber}\left(I+X X^{\star}\right) . \\
& \text { (ii) } \operatorname{ber}^{2}(X) \leqslant \frac{1}{2} \operatorname{ber}\left(X^{\star} X+X X^{\star}\right) .
\end{aligned}
$$

Remark 3.3. Corollary 3.2(ii) is an improvement of (1.1). To see this, note that

$$
\begin{aligned}
\operatorname{ber}^{2}(X) & \leqslant \frac{1}{2} \operatorname{ber}\left(X^{\star} X+X X^{\star}\right) \\
& \leqslant \frac{\operatorname{ber}\left(X^{\star} X\right)+\operatorname{ber}\left(X X^{\star}\right)}{2} \\
& \leqslant \frac{\left\|X^{\star} X\right\|+\left\|X X^{\star}\right\|}{2} \\
& =\|X\|^{2} .
\end{aligned}
$$

In the following theorem, we present some upper bounds of $\operatorname{ber}\left(A^{\star} X B\right)$. To achieve this proposition, we need the next lemma; see [11, p. 265] and [19].

Lemma 3.4. If $X \in \mathbb{B}(\mathscr{H})$ and $x, y \in \mathscr{H}$, then $|\langle X x, y\rangle|^{2} \leqslant\langle|X| x, x\rangle\left\langle\left|X^{\star}\right| y, y\right\rangle$, in which $|X|=\left(X^{\star} X\right)^{\frac{1}{2}}$.

Theorem 3.5. Let $A, B, X \in \mathbb{B}(\mathscr{H})$. Then

$$
\begin{aligned}
& \text { (i) } \operatorname{ber}^{2}\left(A^{\star} X B\right) \leqslant\|X\|^{2} \operatorname{ber}\left(A^{\star} A\right) \operatorname{ber}\left(B^{\star} B\right) . \\
& \text { (ii) } \operatorname{ber}\left(A^{\star} X B\right) \leqslant \frac{1}{2} \operatorname{ber}\left(B^{\star}|X| B+A^{\star}\left|X^{\star}\right| A\right) \text {. }
\end{aligned}
$$


Proof. We have

$$
\begin{aligned}
\left|\left\langle A^{\star} X B \hat{k}_{\lambda}, \hat{k}_{\lambda}\right\rangle\right|^{2} & =\left|\left\langle X B \hat{k}_{\lambda}, A \hat{k}_{\lambda}\right\rangle\right|^{2} \\
& \leqslant\left\|X B \hat{k}_{\lambda}\right\|^{2}\left\|A \hat{k}_{\lambda}\right\|^{2} \\
& \leqslant\|X\|^{2}\left\|B \hat{k}_{\lambda}\right\|^{2}\left\|A \hat{k}_{\lambda}\right\|^{2} \\
& \leqslant\|X\|^{2}\left\langle B \hat{k}_{\lambda}, B \hat{k}_{\lambda}\right\rangle\left\langle A \hat{k}_{\lambda}, A \hat{k}_{\lambda}\right\rangle \\
& =\|X\|^{2}\left\langle B^{\star} B \hat{k}_{\lambda}, \hat{k}_{\lambda}\right\rangle\left\langle A^{\star} A \hat{k}_{\lambda}, \hat{k}_{\lambda}\right\rangle \\
& \leqslant\|X\|^{2} \operatorname{ber}\left(A^{\star} A\right) \operatorname{ber}\left(B^{\star} B\right),
\end{aligned}
$$

whence $\operatorname{ber}^{2}\left(A^{\star} X B\right)=\sup _{\lambda \in \Omega}\left|\left\langle A^{\star} X B \hat{k}_{\lambda}, \hat{k}_{\lambda}\right\rangle\right|^{2} \leqslant\|X\|^{2} \operatorname{ber}\left(A^{\star} A\right) \operatorname{ber}\left(B^{\star} B\right)$, and so we get the first inequality. Also, we have

$$
\begin{aligned}
\left|\left\langle A^{\star} X B \hat{k}_{\lambda}, \hat{k}_{\lambda}\right\rangle\right| & =\left|\left\langle X B \hat{k}_{\lambda}, A \hat{k}_{\lambda}\right\rangle\right| \\
\leqslant & \left\langle|X| B \hat{k}_{\lambda}, B \hat{k}_{\lambda}\right\rangle^{\frac{1}{2}}\left\langle\left|X^{\star}\right| A \hat{k}_{\lambda}, A \hat{k}_{\lambda}\right\rangle^{\frac{1}{2}} \quad \text { (by Lemma 3.4) } \\
\leqslant & \frac{1}{2}\left(\left\langle\left(B^{\star}|X| B\right) \hat{k}_{\lambda}, \hat{k}_{\lambda}\right\rangle+\left\langle\left(A^{\star}\left|X^{\star}\right| A\right) \hat{k}_{\lambda}, \hat{k}_{\lambda}\right\rangle\right) \\
& \quad\left(\text { by the convexity } f(t)=t^{2}\right) \\
= & \frac{1}{2}\left(\left\langle\left(B^{\star}|X| B+A^{\star}\left|X^{\star}\right| A\right) \hat{k}_{\lambda}, \hat{k}_{\lambda}\right\rangle\right) \\
& \leqslant \frac{1}{2} \operatorname{ber}\left(B^{\star}|X| B+A^{\star}\left|X^{\star}\right| A\right) .
\end{aligned}
$$

Hence

$$
\begin{aligned}
\operatorname{ber}\left(A^{\star} X B\right) & =\sup _{\lambda \in \Omega}\left|\left\langle A^{\star} X B \hat{k}_{\lambda}, \hat{k}_{\lambda}\right\rangle\right| \\
& \leqslant \frac{1}{2} \operatorname{ber}\left(B^{\star}|X| B+A^{\star}\left|X^{\star}\right| A\right) .
\end{aligned}
$$

In the special case of Theorem 3.5, for $X=I$ we obtain the next result.

Corollary 3.6. Let $A, B \in \mathbb{B}(\mathscr{H})$. Then
(i) $\operatorname{ber}^{2}\left(A^{\star} B\right) \leqslant \operatorname{ber}\left(A^{\star} A\right) \operatorname{ber}\left(B^{\star} B\right)$.
(ii) $\operatorname{ber}\left(A^{\star} B\right) \leqslant \frac{1}{2} \operatorname{ber}\left(A^{\star} A+B^{\star} B\right)$.

Corollary 3.7. Let $A, B, X \in \mathbb{B}(\mathscr{H})$. Then

$$
\begin{aligned}
& \text { (i) } \operatorname{ber}\left(A^{\star} X B\right) \leqslant \operatorname{ber}^{\frac{1}{2}}\left(B^{\star}|X| B\right) \operatorname{ber}^{\frac{1}{2}}\left(A^{\star}\left|X^{\star}\right| A\right) . \\
& \text { (ii) } \operatorname{ber}\left(A^{\star} X B\right) \leqslant \frac{1}{2} \operatorname{ber}\left(\frac{\|B\|}{\|A\|} B^{\star}|X| B+\frac{\|A\|}{\|B\|} A^{\star}\left|X^{\star}\right| A\right) .
\end{aligned}
$$

Proof. By Theorem 3.5(ii), we have

$$
\begin{aligned}
\operatorname{ber}\left(A^{\star} X B\right) & \leqslant \frac{1}{2} \operatorname{ber}\left(B^{\star}|X| B+A^{\star}\left|X^{\star}\right| A\right) \\
& \leqslant \frac{1}{2}\left(\operatorname{ber}\left(B^{\star}|X| B\right)+\operatorname{ber}\left(A^{\star}\left|X^{\star}\right| A\right)\right) .
\end{aligned}
$$


Now, if we replace $A$ and $B$ by $t A$ and $\frac{1}{t} B(t>0)$ in inequality (3.1), respectively, then we get

$$
\operatorname{ber}\left(A^{\star} X B\right) \leqslant \frac{1}{2}\left(\frac{1}{t^{2}} \operatorname{ber}\left(B^{\star}|X| B\right)+t^{2} \operatorname{ber}\left(A^{\star}\left|X^{\star}\right| A\right)\right) .
$$

Then, from

$$
\inf _{t>0}\left(\frac{1}{t^{2}} \operatorname{ber}\left(B^{\star}|X| B\right)+t^{2} \operatorname{ber}\left(A^{\star}\left|X^{\star}\right| A\right)\right)=2 \operatorname{ber}^{\frac{1}{2}}\left(B^{\star}|X| B\right) \operatorname{ber}^{\frac{1}{2}}\left(A^{\star}\left|X^{\star}\right| A\right),
$$

we get the first inequality. Moreover, if we replace $A$ and $B$ by $\sqrt{\frac{\|A\|}{\|B\|}} A$ and $\sqrt{\frac{\|B\|}{\|A\|}} B$ in Theorem 3.5(ii), respectively, we reach the second inequality.

Using Theorem 3.5, we demonstrate some upper bounds for $\operatorname{ber}\left(A^{\star} X B+B^{\star} Y A\right)$.

Theorem 3.8. Let $A, B, X, Y \in \mathbb{B}(\mathscr{H})$. Then

$$
\begin{aligned}
& \text { (i) } \operatorname{ber}\left(A^{\star} X B+B^{\star} Y A\right) \leqslant \sqrt{2}\left\||X|+\left|Y^{\star}\right|\right\| \operatorname{ber}^{\frac{1}{2}}\left(B^{\star} B\right) \operatorname{ber}^{\frac{1}{2}}\left(A A^{\star}\right) \text {. } \\
& \text { (ii) } \operatorname{ber}\left(A^{\star} X B+B^{\star} Y A\right) \leqslant 2 \sqrt{\|X\|\|Y\|} \operatorname{ber}^{\frac{1}{2}}\left(B^{\star} B\right) \operatorname{ber}^{\frac{1}{2}}\left(A A^{\star}\right) \text {. }
\end{aligned}
$$

Proof. Applying Lemma 2.1 and Theorem 3.5(i), we have

$$
\begin{gathered}
\operatorname{ber}\left(\mathfrak{R e}\left(e^{i \alpha}\left(A^{\star} X B \pm B^{\star} Y A\right)\right)\right)=\operatorname{ber}\left(\mathfrak{R e}\left(A^{\star}\left(e^{i \alpha} X \pm e^{-i \alpha} Y^{\star}\right) B\right)\right) \\
\left(\operatorname{since} \mathfrak{R e}(T)=\mathfrak{R e}\left(T^{\star}\right)\right) \\
\left.\leqslant \operatorname{ber}\left(A^{\star}\left(e^{i \alpha} X \pm e^{-i \alpha} Y^{\star}\right) B\right)\right) \\
(\text { by Lemma } 2.1 \text { for } \theta=0) \\
\leqslant\left\|e^{i \alpha} X \pm e^{-i \alpha} Y^{\star}\right\| \operatorname{ber}^{\frac{1}{2}}\left(B^{\star} B\right) \operatorname{ber}^{\frac{1}{2}}\left(A A^{\star}\right) \\
(\text { by Theorem 3.5(i)). }
\end{gathered}
$$

It follows from the inequalities

$$
\begin{aligned}
\left\|e^{i \alpha} X \pm e^{-i \alpha} Y^{\star}\right\| & =\left\|\left[\begin{array}{cc}
e^{i \alpha} X \pm e^{-i \alpha} Y^{\star} & 0 \\
0 & 0
\end{array}\right]\right\| \\
& =\left\|\left[\begin{array}{cc}
e^{i \alpha} & e^{-i \alpha} \\
0 & 0
\end{array}\right]\left[\begin{array}{cc}
X & 0 \\
\pm Y^{\star} & 0
\end{array}\right]\right\| \\
& \leqslant\left\|\left[\begin{array}{cc}
e^{i \alpha} & e^{-i \alpha} \\
0 & 0
\end{array}\right]\right\|\left\|\left[\begin{array}{cc}
X & 0 \\
\pm Y^{\star} & 0
\end{array}\right]\right\| \\
& =\sqrt{2}\|\|\left[\begin{array}{cc}
X & 0 \\
\pm Y^{\star} & 0
\end{array}\right] \| \\
& =\sqrt{2}\left\|\left(|X|^{2}+\left|Y^{\star}\right|^{2}\right)^{\frac{1}{2}}\right\| \\
& \leqslant \sqrt{2}\left\||X|+\left|Y^{\star}\right|\right\|
\end{aligned}
$$

(applying [3, p. 775] to the function $h(t)=t^{\frac{1}{2}}$ ),

(3.2) and Lemma 2.1 that

$$
\begin{aligned}
\operatorname{ber}\left(A^{\star} X B \pm B^{\star} Y A\right) & =\operatorname{supber}_{\alpha \in \mathbb{R}}\left(\mathfrak{R e}\left(e^{i \alpha}\left(A^{\star} X B \pm B^{\star} Y A\right)\right)\right) \\
& \leqslant \sqrt{2}|||X|+\left|Y^{\star}\right| \| \operatorname{ber}^{\frac{1}{2}}\left(B^{\star} B\right) \operatorname{ber}^{\frac{1}{2}}\left(A A^{\star}\right) .
\end{aligned}
$$


Thus, we get the first inequality. Moreover, using inequality (3.2) we have

$$
\begin{aligned}
\operatorname{ber}\left(\mathfrak{R e}\left(e^{i \alpha}\left(A^{\star} X B \pm B^{\star} Y A\right)\right)\right) & \leqslant\left\|e^{i \alpha} X \pm e^{-i \alpha} Y^{\star}\right\| \operatorname{ber}^{\frac{1}{2}}\left(B^{\star} B\right) \operatorname{ber}^{\frac{1}{2}}\left(A A^{\star}\right) \\
& \leqslant(\|X\|+\|Y\|) \operatorname{ber}^{\frac{1}{2}}\left(B^{\star} B\right) \operatorname{ber}^{\frac{1}{2}}\left(A A^{\star}\right) .
\end{aligned}
$$

Now, if we replace $A$ by $\sqrt{t} A, B$ by $\sqrt{\frac{1}{t}} B, X$ by $t X$ and $Y$ by $\frac{1}{t} Y(t>0)$ in inequality (3.3), then we get

$$
\operatorname{ber}\left(\mathfrak{R e}\left(e^{i \alpha}\left(A^{\star} X B \pm B^{\star} Y A\right)\right)\right) \leqslant\left(t\|X\|+\frac{1}{t}\|Y\|\right) \operatorname{ber}^{\frac{1}{2}}\left(B^{\star} B\right) \operatorname{ber}^{\frac{1}{2}}\left(A A^{\star}\right) .
$$

It follows from $\min _{t>0}\left(t\|X\|+\frac{1}{t}\|Y\|\right)=2 \sqrt{\|X\|\|Y\|}$ and inequality (3.4) that

$$
\begin{aligned}
\operatorname{ber}\left(A^{\star} X B \pm B^{\star} Y A\right) & =\operatorname{supber}_{\alpha \in \mathbb{R}}\left(\mathfrak{R e}\left(e^{i \alpha}\left(A^{\star} X B \pm B^{\star} Y A\right)\right)\right) \\
& \leqslant 2 \sqrt{\|X\|\|Y\| \operatorname{ber}^{\frac{1}{2}}}\left(B^{\star} B\right) \operatorname{ber}^{\frac{1}{2}}\left(A A^{\star}\right) .
\end{aligned}
$$

Hence, we get the second inequality.

Corollary 3.9. If $A, B, X \in \mathbb{B}(\mathscr{H})$, then

$$
\begin{aligned}
& \text { (i) } \operatorname{ber}\left(A^{\star} X \pm X A\right) \leqslant 2\|X\| \operatorname{ber}^{\frac{1}{2}}\left(A A^{\star}\right) . \\
& \text { (ii) } \operatorname{ber}\left(A^{\star} B \pm B^{\star} A\right) \leqslant 2 \operatorname{ber}^{\frac{1}{2}}\left(B^{\star} B\right) \operatorname{ber}^{\frac{1}{2}}\left(A A^{\star}\right) .
\end{aligned}
$$

Proof. If we put $B=I$ and $X=Y$ in Theorem 3.8(ii), then we reach the first inequality and if we take $X=Y=I$ in Theorem 3.8(ii), then we get the second inequality.

\section{Some upper bounds for ber $(A B)-\operatorname{ber}(A) \operatorname{ber}(B)$}

Recall that the Toeplitz operator $T_{\varphi}$ with $\varphi \in L^{\infty}(\partial \mathbb{D})$ is defined on the Hardy space $\mathscr{H}^{2}$ by $T_{\varphi} f=P_{+}(\varphi f)$, where $P_{+}: L^{\infty}(\partial \mathbb{D}) \longrightarrow \mathscr{H}^{2}$ is the Riesz orthogonal projection. It is well known that (see Enliš [6] and Zhu [24]) $\widetilde{T_{\varphi}}(\lambda)=\widetilde{\varphi}(\lambda)(\lambda \in \mathbb{D})$, where $\widetilde{\varphi}$ is the harmonic extension of $\varphi$ into $\mathbb{D}$. Therefore, it is easy to see that

$$
\operatorname{ber}\left(T_{\varphi}\right)=\sup _{\lambda \in \mathbb{D}}|\widetilde{\varphi}(\lambda)|=\sup _{\zeta \in \partial \mathbb{D}}|\varphi(\zeta)|=\|\varphi\|_{\infty}=\left\|T_{\varphi}\right\| .
$$

Then, we have for all $\varphi, \psi \in L^{\infty}(\partial \mathbb{D})$ that

$$
\operatorname{ber}\left(T_{\varphi} T_{\psi}\right) \leq\left\|T_{\varphi} T_{\psi}\right\| \leq\left\|T_{\varphi}\right\|\left\|T_{\psi}\right\|=\|\varphi\|_{\infty}\|\psi\|_{\infty}=\operatorname{ber}\left(T_{\varphi}\right) \operatorname{ber}\left(T_{\psi}\right),
$$

hence

$$
\operatorname{ber}\left(T_{\varphi} T_{\psi}\right) \leq \operatorname{ber}\left(T_{\varphi}\right) \operatorname{ber}\left(T_{\psi}\right) .
$$

Note that Berezin transform is not, in general, multiplicative, i.e., $\widetilde{A B}=\widetilde{A} \widetilde{B}$ (for more information, see Kilič [18]). So, it is natural to ask: when the multiplicative inequality

$$
\operatorname{ber}(A B) \leq \operatorname{ber}(A) \operatorname{ber}(B)
$$

is held?

Our next result proves the inequality $\operatorname{ber}(A B) \leqslant \operatorname{ber}(A) \operatorname{ber}(B)$ for some operators.

Proposition 4.1. Let $A, B \in \mathbb{B}(\mathscr{H})$. If $\overline{\lim }_{\lambda \rightarrow \xi_{0}}\left\|(A-\widetilde{A}(\lambda) I)^{\star} \hat{k}_{\lambda}\right\|=0$ for some $\xi_{0} \in \partial \Omega$, then

$$
\varlimsup_{\lambda \rightarrow \xi_{0}}|\widetilde{A B}(\lambda)| \leqslant \operatorname{ber}(A) \operatorname{ber}(B) .
$$

In particular, if $\varlimsup_{\lambda \rightarrow \xi_{0}}|\widetilde{A B}(\lambda)|=\operatorname{ber}(A B)$, then $\operatorname{ber}(A B) \leqslant \operatorname{ber}(A) \operatorname{ber}(B)$. 
Proof. It follows from $\widetilde{A^{\star}}=\overline{\widetilde{A}}$ that for all $\lambda \in \Omega$ we have

$$
\begin{aligned}
\widetilde{A B}(\lambda) & =\left|\left\langle A B \hat{k}_{\lambda}, \hat{k}_{\lambda}\right\rangle\right| \\
& =\left|\left\langle B \hat{k}_{\lambda}, A^{\star} \hat{k}_{\lambda}\right\rangle\right| \\
& \leq\left|\left\langle B \hat{k}_{\lambda}, A^{\star} \hat{k}_{\lambda}-\widetilde{A^{\star}}(\lambda) \hat{k}_{\lambda}\right\rangle\right|+\left|\left\langle B \hat{k}_{\lambda}, \widetilde{\widetilde{A}}(\lambda) \hat{k}_{\lambda}\right\rangle\right| \\
& \leqslant\|B\|\left\|A^{\star} \hat{k}_{\lambda}-\widetilde{A^{*}}(\lambda) \hat{k}_{\lambda}\right\|+\operatorname{ber}(A) \operatorname{ber}(B),
\end{aligned}
$$

from which, by using the hypotheses of the theorem, we have that there exists a point $\xi_{0} \in \partial \Omega$ such that $\varlimsup_{\lambda \rightarrow \xi_{0}}|\widetilde{A B}(\lambda)| \leqslant \operatorname{ber}(A) \operatorname{ber}(B)$, as desired. The second assertion of the theorem is immediate from the first one.

Note that all Toeplitz operators $T_{\varphi}, \varphi \in L^{\infty}(\partial \mathbb{D})$, on $\mathscr{H}^{2}$ satisfy the conditions

$$
\lim _{r \rightarrow 1^{-}}\left\|T_{\varphi} \hat{k}_{r e e^{i t}}-\widetilde{\varphi}\left(r e^{i t}\right) \hat{k}_{r e}\right\|^{i t} \|_{2}=0
$$

for almost all $t \in[0,2 \pi)$; see Engliš [6]. Also, the operators of the form $T_{\varphi}+K$, where $K$ is compact, satisfy the condition of Proposition 4.1.

Proposition 4.2. If $A, B \in \mathbb{B}(\mathscr{H})$ and $\widetilde{A B}(\lambda) \rightarrow 0$ whenever $\lambda \rightarrow \partial \Omega$, then there exists a point $\lambda_{0} \in \Omega$ such that

$$
\operatorname{ber}(A B)-\operatorname{ber}(A) \operatorname{ber}(B) \leqslant \sqrt{\left.\widetilde{B^{\star} B}\left(\lambda_{0}\right) \widetilde{\left(A A^{\star}\right.}\left(\lambda_{0}\right)-\left|\widetilde{A}\left(\lambda_{0}\right)\right|^{2}\right)}
$$

Proof. By the same argument as in the proof of Proposition 4.1, we have

$$
\begin{aligned}
\widetilde{A B}(\lambda) & \leqslant \operatorname{ber}(A) \operatorname{ber}(B)+\left\|B \hat{k}_{\lambda}\right\|\left\|A^{\star} \hat{k}_{\lambda}-\widetilde{A^{\star}}(\lambda) \hat{k}_{\lambda}\right\| \\
& =\operatorname{ber}(A) \operatorname{ber}(B)+\sqrt{\widetilde{B^{\star} B}(\lambda)\left(\widetilde{A A^{\star}}(\lambda)-|\widetilde{A}(\lambda)|^{2}\right)}
\end{aligned}
$$

for $\lambda \in \Omega$. Since the set $\left\{\left\langle A B \hat{k}_{\lambda}, \hat{k}_{\lambda}\right\rangle: \lambda \in \Omega\right\}$ is bounded, there exists a sequence $\left(\lambda_{n}\right) \subset \Omega$ such that $\operatorname{ber}(A B)=\sup _{\lambda \in \Omega}|\widetilde{A B}(\lambda)|=\lim _{n \rightarrow \infty}\left|\left\langle A B \hat{k}_{\lambda_{n}}, \hat{k}_{\lambda_{n}}\right\rangle\right|$. On the other hand, by the hypotheses $\widetilde{A B}(\lambda) \rightarrow 0$ whenever $\lambda \rightarrow \partial \Omega$, and hence the sequence $\left(\lambda_{n}\right)$ can not approach to the boundary $\partial \Omega$. This shows there exists $\lambda_{0} \in \Omega$ such that $\lim _{n \rightarrow \infty} \lambda_{n}=\lambda_{0}$. Then we obtain from the last inequality that

$$
\operatorname{ber}(A B)-\operatorname{ber}(A) \operatorname{ber}(B) \leqslant \sqrt{\widetilde{B^{\star} B}\left(\lambda_{0}\right)\left(\widetilde{A A^{\star}}\left(\lambda_{0}\right)-\left|\widetilde{A}\left(\lambda_{0}\right)\right|^{2}\right)} .
$$

Recall that the reproducing kernel Hilbert space $\mathscr{H}=\mathscr{H}(\Omega)$ is called standard [20], if $\hat{k}_{\lambda} \rightarrow 0$ weakly whenever $\lambda$ tends to the boundary of $\Omega$. Then $\widetilde{K}(\lambda)$ vanishes on the boundary for any compact operator $K$ on $\mathscr{H}$. This easily shows that if $A$ or $B$ is a compact operator on the standard reproducing kernel Hilbert space $\mathscr{H}$, then $\widetilde{A B}(\lambda) \rightarrow 0$, whenever $\lambda \rightarrow \partial \Omega$, that is $A$ and $B$ satisfy the hypothesis of Proposition 4.2. There are also the following nontrivial examples.

Example 4.3. Let $\theta(z)=\exp \left(\frac{z+1}{z-1}\right)$ be a singular inner function, $T_{\theta}$ be a Toeplitz operator on $\mathscr{H}^{2}$, and let $J$ be an operator (involution) on $\mathscr{H}^{2}$ defined by $(J f)(z):=f(-z)$. Then $\widetilde{J^{\star} T_{\theta}^{\star}(\lambda)} \rightarrow 0$ whenever $\lambda \rightarrow \partial \mathbb{D}$.

Proof. In fact, we have:

$$
\begin{aligned}
\widetilde{J^{\star} T_{\theta}^{\star}(\lambda)} & =\left\langle\widetilde{J^{\star} T_{\theta}^{\star}} \hat{k}_{\lambda}, \hat{k}_{\lambda}\right\rangle=\left(1-|\lambda|^{2}\right)\left\langle\overline{\theta(\lambda)} \frac{1}{1-\bar{\lambda} z}, J \frac{1}{1-\bar{\lambda} z}\right\rangle \\
& =\overline{\theta(\lambda)}\left(1-|\lambda|^{2}\right)\left\langle\frac{1}{1-\bar{\lambda} z}, \frac{1}{1+\bar{\lambda} z}\right\rangle=\overline{\theta(\lambda)} \frac{1-|\lambda|^{2}}{1+|\lambda|^{2}},
\end{aligned}
$$


whence $\widetilde{J^{\star} T_{\theta}^{\star}(\lambda)} \rightarrow 0$, whenever $\lambda \rightarrow \partial \mathbb{D}$. Since $J^{2}=I$, J is invertible on $\mathscr{H}^{2}$ and $T_{\theta}^{\star}$ is not compact on $\mathscr{H}^{2}$, and so $J^{\star} T_{\theta}^{\star}$ is not compact on $\mathscr{H}^{2}$.

There is also an unbounded operator satisfying the hypothesis of Proposition 4.2. Namely, let $D:=\frac{d}{d z}$ denote the differentiation operator on $\mathscr{H}^{2}$, and let $J$ be the same as in Example 4.3. Then we have

$$
\begin{aligned}
\widetilde{J^{\star} D(\lambda)} & =\left\langle J^{\star} D \hat{k}_{\lambda}, \hat{k}_{\lambda}\right\rangle \\
& =\left(1-|\lambda|^{2}\right)\left\langle\frac{d}{d z}\left(\frac{1}{1-\bar{\lambda} z}\right), J \frac{1}{1-\bar{\lambda} z}\right\rangle \\
& =\bar{\lambda}\left(1-|\lambda|^{2}\right)\left\langle\frac{1}{(1-\bar{\lambda} z)^{2}}, \frac{1}{1+\bar{\lambda} z}\right\rangle \\
& =\bar{\lambda} \frac{1-|\lambda|^{2}}{\left(1+|\lambda|^{2}\right)^{2}} \rightarrow 0 \text { as }|\lambda| \rightarrow 1^{-},
\end{aligned}
$$

as desired.

Acknowledgement: We thank to referees for their useful remarks and suggestions which improved the presentation of the paper. The first author would like to thank the Tusi Mathematical Research Group (TMRG). The second author was supported by King Saud University, Deanship of Scientific Research, College of Science Research Center.

\section{References}

[1] Abu-Omar A. and Kittaneh F., Numerical radius inequalities for $n \times n$ operator matrices, Linear Algebra Appl. 468 (2015), $18-26$

[2] Abu-Omar A. and Kittaneh F., Numerical radius inequalities for products and commutators of operators, Houston J. Math. 41 (2015), no. 4, 1163-1173

[3] Ando T. and Zhan X., Norm inequalities related to operator monotone functions, Math. Ann. 315 (1999), no. 4, 771-780.

[4] Berezin F.A., Covariant and contravariant symbols for operators, Math. USSR-Izv. 6 (1972) 1117-1151.

[5] Berezin F.A., Quantization, Math. USSR-Izv. 8 (1974) 1109-1163.

[6] Engli M.š, Toeplitz operators and the Berezin transform on $\mathrm{H}^{2}$, Special issue honoring Miroslav Fiedler and Vlastimil Pták. Linear Algebra Appl. 223/224 (1995), 171-204.

[7] Garayev M.T., Gürdal M. and Okudan A., Hardy-Hilbert's inequality and power inequalities for Berezin numbers of operators, Math. Inequal. App., 19 (2016), no. 3, 883-891

[8] Garayev M.T., Gürdal M., and Saltan S., Hardy type inequality for reproducing kernel Hilbert space operators and related problems, Positivity, 21 (2017), no. 4, 1615-1623.

[9] Gustafson K.E. and Rao D.K.M., Numerical Range, The Field of Values of Linear Operators and Matrices, Springer, New York, 1997.

[10] Hajmohamadi M., Lashkaripour R. and Bakherad M., Improvements of Berezin number inequalities, Linear and Multilinear Algebra, (to appear), doi.org/10.1080/03081087.2018.1538310

[11] Halmos P.R., A Hilbert space problem book, Springer Verlag, New York, 1982.

[12] Karaev M.T., Berezin symbol and invertibility of operators on the functional Hilbert spaces, J. Funct. Anal., 238 (2006) 181192.

[13] Karaev M.T., On the Berezin symbol, J. Math. Sci. (New York) 115 (2003) 2135-2140. Translated from: Zap. Nauchn. Sem. S.-Peterburg. Otdel. Mat. Inst. Steklov. (POMI) 270 (2000) 80-89.

[14] Karaev M.T., Functional analysis proofs of Abel's theorems, Proc. Amer. Math. Soc. 132 (2004) 2327-2329.

[15] Karaev M.T. and Saltan S., Some results on Berezin symbols, Complex Var. Theory Appl. 50 (3) (2005) 185-193.

[16] Karaev M.T., Reproducing kernels and Berezin symbols techniques in various questions of operator theory, Complex Anal. Oper. Theory 7 (2013), no. 4, 983-1018.

[17] Karaev M.T., Saltan S. and Gundogdu D., On the inverse power inequality for the Berezin number of operators, J. Math. Inequal. 12 (2018), no. 4, 997-1003.

[18] Kili S., č, The Berezin symbol and multipliers of functional Hilbert spaces, Proc. Amer. Math. Soc. 123 (1995), no. 12, 36873691.

[19] Kittaneh F., Notes on some inequalitis for Hilbert space operators, Publ. Res. Inst. Math. Sci. 24 (2) (1988), $283-293$.

[20] Nordgren E. and Rosenthal P., Boundary values of Berezin symbols, Oper. Theory Adv. Appl. 73 (1994) 362-368. 
[21] Yamanci U. and Gürdal M., On numerical radius and Berezin number inequalities for reproducing kernel Hilbert space, New York J. Math. 23 (2017), 1531-1537.

[22] Yamanci U., Gürdal M. and Garayev M.T., Berezin number inequality for convex function in reproducing Kernel Hilbert space, Filomat 31 (2017), no. 18, 5711-5717.

[23] Yamazaki T., On upper and lower bounds of the numerical radius and an equality condition, Studia Math. 178 (2007), 83-89.

[24] Zhu K., Operator Theory in Function Spaces, Dekker, New York, 1990. 